\section{RSP}

http://www.rsp.fsp.usp.br/

\title{
Pesquisa Nacional sobre Acesso, Utilização e Promoção do Uso Racional de Medicamentos (PNAUM): avaliação dos serviços de atenção farmacêutica primária
}

\author{
Marco Akerman', Osvaldo de Freitas" \\ Departamento de Política, Gestão e Saúde. Faculdade de Saúde Pública. Universidade de São Paulo. \\ São Paulo, SP, Brasil \\ " Departamento de Ciências Farmacêuticas. Faculdade de Ciências Farmacêuticas. Universidade de São Paulo. \\ Ribeirão Preto, SP, Brasil
}

O uso racional de medicamentos é uma aspiração que não deve ser atribuída somente ao encontro entre profissional de saúde e usuário, pois ultrapassa os limites da mera interação entre prescrição e uso que se dá na relação médico-paciente.

Essa pretensa racionalidade no uso do medicamento clamaria por um difícil alinhamento entre racionalidades distintas dos vários atores que interagem na arena da assistência farmacêutica: governos federal, estaduais e municipais, universidades e cursos técnicos, profissionais de saúde, serviços de saúde públicos e privados, indústria farmacêutica e fornecedores de insumos, usuários e a mídia.

Neste sentido, a máxima "eu penso, logo existo", que indicaria a proeminência do império da razão, poderia falhar, pois "pensamos onde não existimos, ou existimos onde não pensamos" ${ }^{\text {"23 }}$.

Correspondência:

Marco Akerman

Departamento de Política, Gestão e Saúde - FSP-USP

Av. Dr. Arnaldo, 715

01246-903 São Paulo, SP, Brasil

E-mail: marco.akerman@gmail.com

Recebido: 28 abr 2017

Aprovado: 03 mai 2017

Como citar: Akerman M, Freitas O. Pesquisa Nacional sobre Acesso, Utilização e Promoção do Uso Racional de Medicamentos (PNAUM): avaliação dos serviços de atenção farmacêutica primária. Rev Saude Publica. 2017;51 Supl 2:1s.

Copyright: Este é um artigo de acesso aberto distribuído sob os termos da Licença de Atribuição Creative Commons, que permite uso irrestrito, distribuição e reprodução em qualquer meio, desde que o autor e a fonte originais sejam creditados.
Entretanto, isso não nos demoveria da busca da produção de uma visão comum que articulasse os diferentes atores do campo em torno de um conjunto de princípios e acordos que impulsionasse um uso com qualidade dos medicamentos, a saber: (1) fortalecer o direito à saúde por meio de sistemas universais de saúde; (2) prover cuidado resolutivo em consonância com as necessidades da população; (3) estimular procedimentos que sejam efetivos; (4) favorecer a promoção da saúde e a prevenção de doenças; e (5) definir de modo transparente os papéis dos atores no campo.

Com isso, procurar inverter a lógica de que o medicamento seja apenas um "input" na rede de cuidados para adquirir caráter de elemento de uma cadeia interconectada de produção de "outcomes" de qualidades.

Para tanto, se requer: (1) acesso facilitado a medicamentos necessários com custos que a sociedade possa suportar; (2) medicamentos que atendam a critérios de qualidade, segurança e eficácia; (3) uso com qualidade dos medicamentos; (4) estímulo a segmentos da indústria que sejam viáveis e responsáveis.

Diante desse contexto, governos e universidades se reuniram em torno da Pesquisa Nacional sobre Acesso, Utilização e Promoção do Uso Racional de Medicamentos (PNAUM) para produzir um amplo retrato da situação do acesso e do uso do medicamento no Brasil ${ }^{12}$.

A Revista de Saúde Pública publicou, em suplemento anterior ${ }^{6}$, resultados do inquérito domiciliar abrangente da PNAUM, que coletou informações preciosas em relação a vários 
aspectos do acesso e uso de medicamentos: na infância; entre os idosos; para doenças crônicas; de fornecimento gratuito; para tratamento da hipertensão arterial; e genéricos. Também foram investigados os gastos catastróficos em saúde, a automedicação, os fatores que influenciam a adesão ao tratamento e a prescrição de contraceptivos.

O presente Suplemento se debruça sobre a avaliação dos serviços de assistência farmacêutica primários - indicando avanços e desafios -, os métodos utilizados, as concepções do que se entende por assistência farmacêutica, as atividades farmacêuticas clínicas realizadas, a maneira como a assistência farmacêutica vem se institucionalizando nos municípios e como o acesso a medicamentos ocorre. Ainda, este suplemento explora como são organizadas a força de trabalho, a seleção, a disponibilidade e a dispensação de medicamentos essenciais na atenção básica, bem como o financiamento e a gestão da assistência farmacêutica, e explora aspectos relacionados com os usuários - acesso, utilização, uso racional, polifarmácia, satisfação e qualidade de vida.

Costa et al..$^{13}$ sintetizam os principais resultados com base em narrativa crítica dos elementos das políticas farmacêuticas no Brasil. Apesar dos avanços, fruto do empenho do conjunto de atores implicados, os resultados da pesquisa apontam desafios, como o acesso equitativo dos medicamentos, a estruturação dos serviços farmacêuticos, o aprimoramento da logística e da gestão e a implantação de ações voltadas ao cuidado farmacêutico nas unidades de saúde. Já Álvares et al. ${ }^{1}$ descrevem de maneira clara, objetiva e detalhada o planejamento, a metodologia utilizada na Pesquisa Nacional sobre Acesso, Utilização e Promoção do Uso Racional de Medicamentos, desde o cálculo amostral e atores envolvidos até a operacionalização em campo.

Embora haja movimentos na gestão do Sistema Único de Saúde (SUS) para a criação de algum grau de padronização de procedimentos, Costa et al. ${ }^{10}$ mostram uma grande diversidade de entendimentos da assistência farmacêutica por parte dos atores relacionados a ela. Porém, foi identificado, no processo de sua reorientação, um movimento que reflete uma mudança gradual do paradigma técnico, centrado na logística de medicamentos, para uma abordagem orientada ao usuário dos serviços de saúde.

Entretanto, Araújo et al. ${ }^{3}$ informam que ainda são incipientes as atividades de natureza clínica desempenhadas por farmacêuticos no Brasil. Isso pode ser atribuído à falta de local adequado e à pequena participação em atividades educativas de promoção da saúde, sugerindo limitada integração dos farmacêuticos na equipe de saúde e da assistência farmacêutica nas demais ações de saúde.

Souza et al..$^{25}$ caracterizam o estágio atual da institucionalização da assistência farmacêutica nos municípios brasileiros, que é heterogêneo e parcial, revelando desigualdades regionais. Por isso, Barros et al. ${ }^{5}$ mostram que o acesso a medicamentos foi maior em locais onde estavam presentes aspectos das seguintes dimensões: ferramentas de gestão; participação e controle social; financiamento e estrutura.

E como os medicamentos são selecionados para a rede de serviços? Karnikowski et al. ${ }^{17}$ apresentam a primeira pesquisa nacional de caracterização do processo de seleção de medicamentos no âmbito da atenção primária à saúde. Todos os atores entrevistados afirmaram possuir uma lista de medicamentos essenciais, mas que atende apenas parcialmente as demandas de saúde. Por conseguinte, Nascimento et al. ${ }^{21}$ mostram que, apesar dos esforços, ainda é um desafio assegurar o acesso aos medicamentos essenciais no âmbito da atenção primária do SUS e apresenta subsídios para o aprimoramento da assistência farmacêutica na rede pública.

Além disso, Leite et al..$^{18}$ constatam que há grandes diferenças de modelos de organização da dispensação. A centralização da dispensação de medicamentos em farmácias isoladas dos serviços de saúde está associada a melhores condições estruturais e de profissionais, assim como nas unidades dispensadoras das regiões Sul, Sudeste e Centro-Oeste. No entanto, o desenvolvimento da dispensação como serviço de saúde ainda não prevalece em qualquer tipo de farmácia ou região do país. 
Independentemente das características na seleção e dispensação, Costa et al. ${ }^{11}$ mostram que a situação sanitária dos medicamentos na atenção básica no Brasil é preocupante, face ao descumprimento da legislação sanitária específica para os estabelecimentos de dispensação. Os autores também descrevem um amplo conjunto de requisitos imprescindíveis à conservação dos medicamentos e um descompasso entre os esforços para promover o acesso e a organização e qualificação dos serviços farmacêuticos. Nesse sentido, Leite et al. ${ }^{19}$ sugerem que a ambiência dos serviços de farmácia precisa ser reestruturada de modo a possibilitar a humanização do atendimento e melhores condições de trabalho aos profissionais.

Sem recursos financeiros adequados não há como aperfeiçoar os aspectos de seleção e dispensação e a qualidade de conservação e estoque dos medicamentos. Faleiros et al. ${ }^{14}$ discutem o financiamento da assistência farmacêutica e levantam os fatores relacionados ao financiamento do Componente Básico da Assistência Farmacêutica no âmbito da gestão municipal do SUS, em 600 municípios distribuídos nas cinco regiões do país. Os resultados mostram graves deficiências na gestão e insuficiência dos recursos destinados ao Componente Básico da Assistência Farmacêutica e a exaustão do modelo de financiamento. A gestão também é abordada por Gerlack et al..$^{15}$ ao identificarem que, infelizmente, as normativas ditadas tanto pelo arcabouço legal quanto pelo político da assistência farmacêutica não são cumpridas.

E como os profissionais de saúde se movem frente a esse perfil estabelecido até aqui? Carvalho et al. ${ }^{7}$ observam importantes deficiências na composição da força de trabalho nas unidades de dispensação que devem comprometer a qualidade do uso dos medicamentos e seus resultados na saúde da população.

Já os usuários, razão maior dos serviços de saúde, são tratados sob vários aspectos.

Foram entrevistados 8.803 usuários distribuídos nas diferentes regiões do país ${ }^{16}$. A maioria eram mulheres (75,8\%). Metade dos usuários foi classificada como classe C e 24,8\% recebem bolsa-família. Apenas 9,8\% possuem plano de saúde, com maior proporção na região Sul e menor na Norte e Centro-Oeste. Foi entre os homens que o consumo de bebida alcoólica e o tabagismo estavam mais presentes. Costa e Silveira ${ }^{9}$ aprofundaram a análise e notaram que $76,2 \%$ dos usuários relataram ter utilizado medicamentos (média de 2,3) no período estipulado. No geral, foram pessoas com baixa escolaridade e com comorbidades, especialmente entre os idosos (65 anos ou mais). Este grupo também relata maior dificuldade no uso de medicamento, tornando-os mais vulneráveis.

Nascimento et al..$^{22}$ identificaram que a polifarmácia (uso de cinco ou mais medicamentos) é uma realidade na população atendida no âmbito da atenção primária do SUS e pode estar relacionada ao uso exacerbado ou inapropriado de medicamentos.

Na perspectiva do usuário, Álvares et al. ${ }^{2}$ comparam os resultados da avaliação do acesso aos medicamentos na atenção primária do SUS compatível com países desenvolvidos. No entanto, o acesso continua sendo um desafio, pois ainda é fortemente comprometido pela baixa disponibilidade de medicamentos essenciais em unidades públicas de saúde.

Soeiro et al. ${ }^{24}$ mostraram a satisfação do usuário com a assistência farmacêutica: o percentual geral foi de 58,4\% (IC95\% 54,4-62,3), com destaque para a dimensão aspectos interpessoais 90,5\% (IC95\% 88,9-91,8).

Para além do uso do medicamento, Ascef et al. ${ }^{4}$ analisam a qualidade de vida dos usuários da atenção primária à saúde, discutem os fatores predominantes e sugerem que este indicador pode nortear a integralidade do cuidado e ações de promoção da saúde.

O artigo de Lima et al..$^{20}$ finaliza o suplemento e mostra que os resultados da avaliação dos indicadores relacionados ao uso racional de medicamentos e seus fatores associados nas unidades básicas de saúde mostram variações significativas entre as regiões do Brasil. 


\section{REFERÊNCIAS}

1. Álvares J, Alves MCGP, Escuder MML, Almeida AM, Izidoro JB, Guerra Junior AA, et al. Pesquisa Nacional sobre Acesso, Utilização e Promoção do Uso Racional de Medicamentos: métodos. Rev Saude Publica. 2017;51 Supl 2:4s. https://doi.org/10.11606/S1518-8787.2017051007027

2. Álvares J, Guerra Junior AA, Araújo VE, Almeida AM, Dias CZ, Oliveira BA, et al. Acesso aos medicamentos pelos usuários da atenção primária no Sistema Único de Saúde. Rev Saude Publica. 2017;51 Supl 2:20s. https://doi.org/10.11606/S1518-8787.2017051007139

3. Araújo PS, Costa EA, Guerra Junior AA, Acurcio FA, Guibu IA, Álvares J, et al. Atividades farmacêuticas de natureza clínica na atenção básica no Brasil. Rev Saude Publica. 2017;51 Supl 2:6s. https://doi.org/10.11606/S1518-8787.2017051007109

4. Ascef BO, Haddad JPA, Álvares J, Guerra Junior AA, Costa EA, Acurcio FA, et al. Qualidade de vida relacionada à saúde dos usuários da atenção primária no Brasil. Rev Saude Publica. 2017;51 Supl 2:22s. https://doi.org/10.11606/S1518-8787.2017051007134

5. Barros RD, Costa EA, Santos DB, Souza GS, Álvares J, Guerra Junior AA, et al. Acesso a medicamentos: relações com a institucionalização da assistência farmacêutica. Rev Saude Publica. 2017;51 Supl 2:8s. https://doi.org/10.11606/S1518-8787.2017051007138

6. Bermudez JAZ, Barros MBA. Perfil do acesso e da utilização de medicamentos da população brasileira: contribuições e desafios da PNAUM - Inquérito Domiciliar. Rev Saude Publica. 2016;50 Supl 2:2s. https://doi.org/10.1590/S1518-8787.201605000supl2ap

7. Carvalho MN, Álvares J, Costa KS, Guerra Junior AA, Acurcio FA, Costa EA, et al. Força de trabalho na assistência farmacêutica da atenção básica do SUS, Brasil. Rev Saude Publica. 2017;51 Supl 2:16s. https://doi.org/10.11606/S1518-8787.2017051007110

8. Commonwealth of Australia, Department of Health and Ageing. National Medicines Policy. Canberra; 1999.

9. Costa CMF, Silveira MR. Utilização de medicamento pelos usuários da atenção primária do Sistema Único de Saúde. Rev Saude Publica. 2017;51 Supl 2:18s. https://doi.org/10.11606/S1518-8787.2017051007144

10. Costa EA, Araújo PS, Penaforte TR, Barreto JL, Guerra Junior AA, Acurcio FA, Guibu IA, et al. Concepções de assistência farmacêutica na atenção primária à saúde, Brasil. Rev Saude Publica. 2017;51 Supl 2:5s. https://doi.org/10.11606/S1518-8787.2017051007107

11. Costa EA, Araújo PS, Pereira MT, Souto AC, Souza GS, Guerra Junior AA, et al. Situação sanitária dos medicamentos na atenção básica no Sistema Único de Saúde. Rev Saude Publica. 2017;51 Supl 2:12s. https://doi.org/10.11606/S1518-8787.2017051007106

12. Costa KS, Tavares NUL, Nascimento Júnior JM, Mengue SS, Álvares J, Guerra Junior AA, et al. Assistência farmacêutica na atenção primária: a pactuação interfederativa no desenvolvimento das políticas farmacêuticas no Sistema Único de Saúde (SUS). Rev Saude Publica. 2017;51 Supl 2:2s. https://doi.org/10.11606/S1518-8787.201705100supl2ap

13. Costa KS, Tavares NUL, Nascimento Júnior JM, Mengue SS, Álvares J, Guerra Junior AA, et al. Avanços e desafios da assistência farmacêutica na atenção primária no Sistema Único de Saúde. Rev Saude Publica. 2017;51 Supl 2:3s. https://doi.org/10.11606/S1518-8787.2017051007146

14. Faleiros DR, Acurcio FA, Álvares J, Nascimento RCRM, Costa EA, Guibu IA, et al. Financiamento da assistência farmacêutica na gestão municipal do Sistema Único de Saúde. Rev Saude Publica. 2017;51 Supl 2:14s. https://doi.org/10.11606/S1518-8787.2017051007060

15. Gerlack LF, Karnikowski MGA, Areda CA, Galato D, Oliveira AG, Álvares J, et al. Gestão da assistência farmacêutica na atenção primária no Brasil. Rev Saude Publica. 2017;51 Supl 2:15s. https://doi.org/10.11606/S1518-8787.2017051007063

16. Guibu IA, Moraes JC, Guerra Junior AA, Costa EA, Acurcio FA, Costa KS, et al. Características principais dos usuários dos serviços de atenção primária à saúde no Brasil. Rev Saude Publica. 2017;51 Supl 2:17s. https://doi.org/10.11606/S1518-8787.2017051007070

17. Karnikowski MGO, Galato D, Meiners MMMA, Silva EV, Gerlack LF, Bós AJG, et al. Caracterização da seleção de medicamentos para a atenção primária no Brasil. Rev Saude Publica. 2017;51 Supl 2:9s. https://doi.org/10.11606/S1518-8787.2017051007065

18. Leite SN, Bernardo NLMC, Álvares J, Guerra Junior AA, Costa EA, Acurcio FA, et al. Serviço de dispensação de medicamentos na atenção básica no SUS. Rev Saude Publica. 2017;51 Supl 2:11s. https://doi.org/10.11606/S1518-8787.2017051007121 
19. Leite SN, Manzini F, Álvares J, Guerra Junior AA, Costa EA, Acurcio FA, et al. Infraestrutura das farmácias da atenção básica no Sistema Único de Saúde: análise dos dados da PNAUM-Serviços. Rev Saude Publica. 2017;51 Supl 2:13s. https://doi.org/10.11606/S1518-8787.2017051007120

20. Lima MG, Álvares J, Guerra Junior AA, Costa EA, Guibu IA, Soeiro OM, et al. Indicadores relacionados ao uso racional de medicamentos e seus fatores associados. Rev Saude Publica. 2017;51 Supl 2:23s. https://doi.org/10.11606/S1518-8787.2017051007137

21. Nascimento RCRM, Álvares J, Guerra Junior AA, Gomes IC, Costa EA, Leite SN, et al. Disponibilidade de medicamentos essenciais na atenção primária do Sistema Único de Saúde. Rev Saude Publica. 2017;51 Supl 2:10s. https://doi.org/10.11606/S1518-8787.2017051007062

22. Nascimento RCRM, Álvares J, Guerra Junior AA, Gomes IC, Silveira MR, Costa EA, et al. Polifarmácia: uma realidade na atenção primária do Sistema Único de Saúde. Rev Saude Publica. 2017;51 Supl 2:19s. https://doi.org/10.11606/S1518-8787.2017051007136

23. Safatle V. Há coisas que não conseguiremos falar sem mudarmos a maneira de dizê-las. Folha de São Paulo. 02 dez 2016 [citado 29 abr 2017]. Disponível em: http://www1.folha.uol. com.br/colunas/vladimirsafatle/2016/12/1837509-ha-coisas-que-nao-conseguiremos-falar-semmudarmos-a-maneira-de-dize-las.shtml

24. Soeiro OM, Tavares NUL, Nascimento Júnior JM, Guerra Junior AA, Costa EA, Acurcio FA, et al. Satisfação dos usuários com os serviços da assistência farmacêutica na atenção básica nos municípios brasileiros. Rev Saude Publica. 2017;51 Supl 2:21s. https://doi.org/10.11606/S1518-8787.2017051007145

25. Souza GS, Costa EA, Barros RD, Pereira MT, Barreto JL, Guerra Junior AA, et al. Caracterização da institucionalização da assistência farmacêutica na atenção básica no Brasil. Rev Saude Publica. 2017;51 Supl 2:7s. https://doi.org/10.11606/S1518-8787.2017051007135 